\title{
Physical security layer with friendly jammer in half-duplex relaying networks over rayleigh fading channel: Intercept probability analysis
}

\author{
Phu Tran Tin ${ }^{1}$, Duy Hung $\mathrm{Ha}^{2}$, Minh Tran ${ }^{3}$, Tran Thanh Trang ${ }^{4}$ \\ ${ }^{1}$ Faculty of Electronics Technology, Industrial University of Ho Chi Minh City, Vietnam \\ ${ }^{2}$ Wireless Communications Research Group, Faculty of Electrical and Electronics Engineering, \\ Ton Duc Thang University, Vietnam \\ ${ }^{3}$ Optoelectronics Research Group, Faculty of Electrical and Electronics Engineering, Ton Duc Thang University, Vietnam \\ ${ }^{4}$ National Key Laboratory of Digital Control and System Engineering, Vietnam
}

\begin{tabular}{l} 
Article Info \\
\hline Article history: \\
Received Dec 10, 2019 \\
Revised Feb 17, 2020 \\
Accepted Mar 23, 2020 \\
\hline
\end{tabular}

Keywords:

Energy harvesting

Half-duplex

Intercept probability

Monte Carlo Simulation

Relaying network

\begin{abstract}
In this research, thephysical security layer with a friendly jammerin half-duplex (HD) relaying networksover the Rayleigh fading channel is proposed and investigated. Firstly, we proposed the system model and the time switching,power splitting protocolsfor the system model. Then we conductedthe mathematical analysisfor deriving theexact analysis and asymptotic analysisintegral forms for intercept probability (IP). Finally, the analytical formulationis verified by the Monte Carlo Simulation with all main system parameters. From the results, we can show that the simulation and analytical values are the same values.
\end{abstract}

This is an open access article under the CC BY-SAlicense.

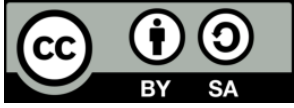

\section{Corresponding Author:}

Duy Hung Ha,

Wireless Communications Research Group,

Faculty of Electrical and Electronics Engineering,

Ton Duc Thang University,

Ho Chi Minh City, Vietnam.

Email: haduyhung@tdtu.edu.vn

\section{INTRODUCTION}

Nowadays, harvesting energy (EH) from green environmental sources such as sun, wind, etc and converting this energy into the electrical energy for the communication network devices is the hot reseach direction in both civil and industial purposes. In the series of the leading environmental green energy sources, the radio frequency (RF) signals can be considered as the prospective energy source in the future. $\mathrm{RF}$ signals can be proposed as the role of information transmission or harvesting energy in the sensor nodes [1-8]. In the past, we used the traditional network security protocol, which is working based on cryptographic technologies for security protecting wireless networks. For extensive research efforts, the physical-layer security (PLS), which is opareting normal wihthout key negotiation and distribution, is proposed and investigated in the modern communication network. Furthermore, PLS technology has a huge advantage for protecting the confidential communication between a source node to its destination as studied [9-10]. The PLS in the wireless networks with cooperative relaying, jamming, multiuser scheduling, multiple-input-multiple-output (MIMO) ..etc have been proposed and investigated in [11-15]. 
In this research, the physical security layer with a friendly jammer in half-duplex (HD) relaying networks over the Rayleigh fading channel is proposed and investigated. In the first stage, we proposed the system model and the time switching, power splitting protocols for the system model. Then we conducted the mathematical analysis for deriving the exact analysis and asymptotic analysis integral forms for intercept probability (IP). Finally, the analytical formulation is verified by the Monte Carlo Simulation with all main system parameters. The research results show that the analytical and simulation are the same values.

\section{SYSTEM MODEL}

The system model, in which a source node (S) communicates with a destination node (D) via the intermediate Relay node $(\mathrm{R})$ in the presence of a passive eavesdropper $(\mathrm{E})$ with the help of a friendly jammer $(\mathrm{J})$, is illustrated in Figure 1. The energy harvesting (EH) and information processing (IT) processes of the system model are drwan in Figure 2. In this scheme, $T$ is the block time in which the source fully transmits the information data to the destination. In the first interval time $(\alpha \mathrm{T})$, the $\mathrm{R}$ and $\mathrm{J}$ harvest energy from the $\mathrm{S}$ signal, where $\alpha$ is the time switching factor $\alpha \in(0,1)$ In the two remaining intervals time $(1-\alpha) \mathrm{T} / 2$, the $\mathrm{S}$ and $\mathrm{R}$ node transfer information to the D node [5-7, 16-24].

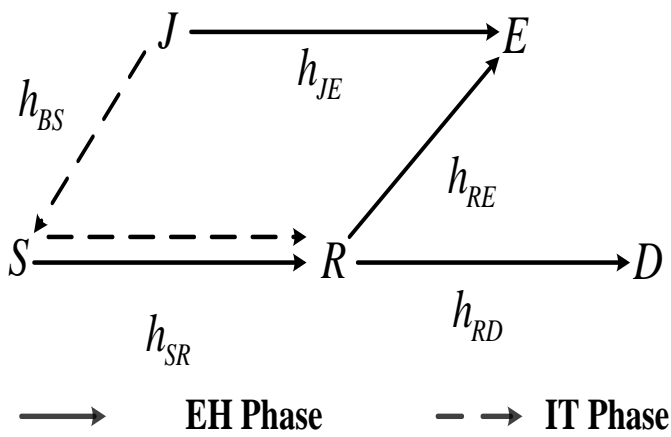

Figure 1. System model

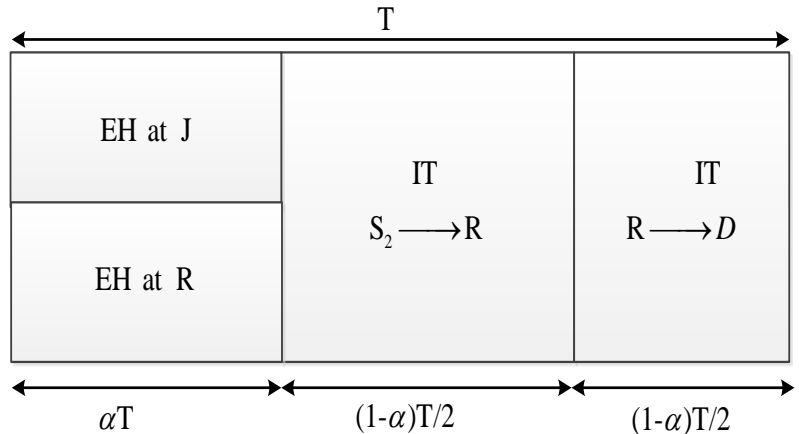

Figure 2. The EH and IT phases

\subsection{Energy harvesting (EH) phase}

In the first phase, the source will supply the energy for both jammer and relay nodes. Hence, the harvested energy at the jammer and relay can be given as, respectively:

$$
\begin{aligned}
& E_{J}=\eta P_{S} \alpha T\left|h_{S J}\right|^{2} \\
& E_{R}=\eta P_{S} \alpha T\left|h_{S R}\right|^{2}
\end{aligned}
$$

where $0<\eta \leq 1$ is energy conversion efficiency, $\mathrm{P}_{\mathrm{S}}$ is the average transmitted power at the source, and $\mathrm{h}_{\mathrm{SJ}}, \mathrm{h}_{\mathrm{SR}}$ are the channel gain of S-J link, S-R link, respectively.

The average transmitted power at the jammer and relay nodes can be obtained from(1) and (2), respectively:

$$
\begin{aligned}
& P_{J}=\frac{E_{J}}{(1-\alpha) T / 2}=\frac{\eta P_{S} \alpha T\left|h_{S J}\right|^{2}}{(1-\alpha) T / 2}=\kappa P_{S}\left|h_{S J}\right|^{2} \\
& P_{R}=\kappa P_{S}\left|h_{S R}\right|^{2}
\end{aligned}
$$

where $\kappa=\frac{2 \eta \alpha}{1-\alpha}$ 


\subsection{Information transmission phase}

In the second phase, the received signal at the $\mathrm{R}$ can be rewritten as:

$$
y_{r}=h_{S R} x_{s}+n_{r}
$$

whereh $_{\mathrm{SR}}$ is the channel gain of $\mathrm{S}-\mathrm{R}$ link, $\mathrm{x}_{\mathrm{S}}$ is the transmitted signal from the $\mathrm{S}$ and $\mathrm{n}_{\mathrm{r}}$ is additive white Gaussian noise (AWGN) with variance $\mathrm{N}_{0}$.

In the third phase, the received signal at the destination can be given by:

$$
y_{d}=h_{R D} x_{r}+n_{d}
$$

whereh $\mathrm{RD}_{\mathrm{D}}$ is the channel gain of R-D link, $\mathrm{x}_{\mathrm{r}}$ is the transmitted signal from relay and $\mathrm{n}_{\mathrm{d}}$ is (AWGN) with variance $\mathrm{N}_{0}$. given as:

Here, we consider amplify and forward (AF) mode at the relay. Hence, the amplifying factor can be

$$
\zeta=\frac{x_{r}}{y_{r}}=\sqrt{\frac{P_{R}}{P_{s}\left|h_{S R}\right|^{2}+N_{0}}}
$$

The received signal at the E can be given by:

$$
\begin{aligned}
y_{E} & =h_{R E} x_{r}+h_{J E} x_{J}+n_{E}=h_{R E} \beta y_{r}+h_{J E} x_{J}+n_{E} \\
& =h_{R E} \beta\left[h_{S R} x_{s}+n_{r}\right]+h_{J E} x_{J}+n_{E} \\
& =\underbrace{h_{R E} \beta h_{S R} x_{s}}_{\text {signal }}+\underbrace{h_{R E} \beta n_{r}+h_{J E} x_{J}+n_{E}}_{\text {noise }}
\end{aligned}
$$

whereh $h_{R E}$ is the channel gain of R-E link and $n_{E}$ is $A W G N$ with variance $N_{0}$.

\section{INTERCEPT PROBABILITY (IP)}

The SNR at the eavesdropper can be expressed as:

$$
\gamma_{E}=\frac{\mathrm{E}\left\{\mid \text { signal }\left.\right|^{2}\right\}}{\mathrm{E}\left\{\mid \text { noise }\left.\right|^{2}\right\}}=\frac{\left|h_{S R}\right|^{2}\left|h_{R E}\right|^{2} \beta^{2} P_{s}}{\left|h_{R E}\right|^{2} \beta^{2} N_{0}+\left|h_{J E}\right|^{2} P_{J}+N_{0}}
$$

Substituting (3) into (9), we have:

$$
\gamma_{E}=\frac{\left|h_{S R}\right|^{2}\left|h_{R E}\right|^{2} \psi}{\left|h_{R E}\right|^{2}+\frac{\kappa \psi\left|h_{S J}\right|^{2}\left|h_{J E}\right|^{2}+1}{\beta^{2}}}
$$

where $\psi=\frac{P_{s}}{N_{0}}$

After doing some algebra, the (10) can be reformulated as:

$$
\begin{aligned}
\gamma_{E} & =\frac{\left|h_{S R}\right|^{4}\left|h_{R E}\right|^{2} \kappa \psi}{\kappa\left|h_{S R}\right|^{2}\left|h_{R E}\right|^{2}+\kappa \psi\left|h_{S R}\right|^{2}\left|h_{S J}\right|^{2}\left|h_{J E}\right|^{2}+\kappa\left|h_{S J}\right|^{2}\left|h_{J E}\right|^{2}+1} \\
& =\frac{\kappa \psi X^{2} Y}{\kappa X Y+\kappa \psi X Z+\kappa Z+1}
\end{aligned}
$$


where $X=\left|h_{S R}\right|^{2}, Y=\left|h_{R E}\right|^{2}, Z=\left|h_{S J}\right|^{2}\left|h_{J E}\right|^{2}$

Lemmal.Please note that all channels are the Rayleigh fading channels, so the probability density function (PDF) of $\left|h_{i}\right|^{2}$ can be given by:

$$
f_{\left|h_{i}\right|^{2}}(x)=\lambda_{i} e^{-\lambda_{i} x}
$$

where $i \in(S R, R E)$. Moreover, the cumulative distribution function (CDF) of $\left|h_{i}\right|^{2}$ also can be obtained by:

$$
F_{\left|h_{i}\right|^{2}}(x)=1-e^{-\lambda_{i} x}
$$

where $\lambda_{i}$ is the mean value of the exponential random variable $\left|h_{i}\right|^{2}$.

Lemma2.The CDF of $Z$ can be computed as respectively:

$$
F_{Z}(a)=\int_{0}^{\infty} F_{\left|h_{S j}\right|^{2}}\left(\left.\frac{a}{\left|h_{J E}\right|^{2}}|| h_{J E}\right|^{2}=x\right) f_{\left|h_{J E}\right|^{2}}(x) d x
$$

Utilizing the result in [25], the $\mathrm{CDF}$ of $\mathrm{Z}$ can be shown as the below equation, respectively:

$$
F_{Z}(a)=1-2 \sqrt{\lambda_{S J} \lambda_{J E} a} K_{1}\left(2 \sqrt{\lambda_{S J} \lambda_{J E} a}\right)
$$

where $K_{v}(\bullet)$ is the modified Bessel function of the second kind and $\mathrm{v}^{\text {th }}$ order and $\lambda_{S J}, \lambda_{J E}$ are mean of the random variable (RV) $\left|h_{S J}\right|^{2},\left|h_{J E}\right|^{2}$, respectively.

From (15), the PDF of $\mathrm{Z}$ can be calculated as, after applying the formula,

$$
\begin{aligned}
& \frac{\partial K_{n}(z)}{\partial z}=-K_{n-1}(z)-\frac{n}{z} K_{n}(z) \\
& f_{Z}(a)=\frac{\partial F_{Z}(a)}{\partial a}=2 \sqrt{\lambda_{S J} \lambda_{J E}} K_{0}\left(2 \sqrt{\lambda_{S J} \lambda_{J E} a}\right)
\end{aligned}
$$

\subsection{Exact analysis}

The IP can be defined by:

$$
I P=\operatorname{Pr}\left(\gamma_{E} \geq \gamma_{t h}\right)
$$

Substituting (11) into (17); finally, we have:

$$
\begin{aligned}
I P & =\operatorname{Pr}\left(\frac{\kappa \psi X^{2} Y}{\kappa X Y+\kappa \psi X Z+\kappa Z+1} \geq \gamma_{t h}\right) \\
& =\operatorname{Pr}\left\{Z\left(\kappa \psi \gamma_{t h} X+\kappa \gamma_{t h}\right) \leq X Y\left(\kappa \psi X-\kappa \gamma_{t h}\right)-\gamma_{t h}\right\} \\
& =\left\{\begin{array}{rr}
\operatorname{Pr}\left\{Z \leq \frac{X Y\left(\kappa \psi X-\gamma_{t h}\right)-\gamma_{t h}}{\left(\kappa \psi \gamma_{t h} X+\kappa \gamma_{t h}\right)},\right. & \left.X \geq \frac{\gamma_{t h}}{\psi} \text { and } X Y \geq \frac{\gamma_{t h}}{\left(\kappa \psi X-\kappa \gamma_{t h}\right)}\right\} \\
0 \quad, X<\frac{\gamma_{t h}}{\psi} \text { or } X Y<\frac{\gamma_{t h}}{\left(\kappa \psi X-\kappa \gamma_{t h}\right)}
\end{array}\right.
\end{aligned}
$$

where $\gamma_{t h}=2^{2 R}-1$ is the threshold of system and $\mathrm{R}$ : target rate.

The (20) can be rewritten by: 


$$
\begin{aligned}
I P & =\operatorname{Pr}\left\{\begin{array}{l}
X \geq \frac{\gamma_{t h}}{\psi} \\
Y \geq \frac{\gamma_{t h}}{X\left(\kappa \psi X-\kappa \gamma_{t h}\right)} \\
Z \leq \frac{X Y\left(\kappa \psi X-\kappa \gamma_{t h}\right)-\gamma_{t h}}{\left(\kappa \psi \gamma_{t h} X+\kappa \gamma_{t h}\right)} \\
=\int_{\frac{\gamma_{t h}}{\psi}}^{\infty} f_{X}(x) d x \int_{\frac{\gamma_{t h}}{x\left(\kappa \psi x-\kappa \gamma_{t h}\right)}}^{\infty} f_{Y}(y) d y \int_{0}^{g(x, y)} f_{Z}(z) d z
\end{array}\right.
\end{aligned}
$$

where $g(x, y)=\frac{x y\left(\kappa \psi x-\kappa \gamma_{t h}\right)-\gamma_{t h}}{\left(\kappa \psi \gamma_{t h} x+\kappa \gamma_{t h}\right)}$

Applying (16) and (19) can be reformulated as:

$$
I P=2 \lambda_{S R} \lambda_{R E} \sqrt{\lambda_{S J} \lambda_{J E}} \int_{\frac{\gamma_{h h}}{\kappa \psi}}^{\infty} \exp \left(-\lambda_{S R} x\right) d x \int_{\frac{\gamma_{h h}}{x\left(\kappa \psi x-\gamma_{h h}\right)}}^{\infty} \exp \left(-\lambda_{R E} y\right) d y \int_{0}^{g(x, y)} K_{0}\left(2 \sqrt{\lambda_{S J} \lambda_{J E} z}\right) d z
$$

\subsection{Asymptotic analysis}

Atthe high SNRregime, the (11)can be approximatedas $\gamma_{E}^{\infty} \approx \frac{X Y}{Z}$

The IP can be computed as:

$I P^{\infty}=\operatorname{Pr}\left(\gamma_{E}^{\infty} \geq \gamma_{t h}\right)=\operatorname{Pr}\left\{\frac{X Y}{Z} \geq \gamma_{t h}\right\}=\operatorname{Pr}\left\{Z \leq \frac{W}{\gamma_{t h}}\right\}=\int_{0}^{\frac{w}{\gamma_{t h}}} F_{Z}\left(\frac{W}{\gamma_{t h}} \mid W=w\right) f_{W}(w) d w$

where $W=X Y$

Similar in Lemma2,the CDF and PDF ofW can be obtained as, respectively:

$$
\begin{aligned}
& F_{W}(b)=1-2 \sqrt{\lambda_{S R} \lambda_{R E} b} K_{1}\left(2 \sqrt{\lambda_{S R} \lambda_{R E} b}\right) \\
& f_{W}(b)=2 \sqrt{\lambda_{S R} \lambda_{R E}} K_{0}\left(2 \sqrt{\lambda_{S R} \lambda_{R E} b}\right)
\end{aligned}
$$

Substituting (15) and(23) into (21), we have:

$$
I P^{\infty}=2 \sqrt{\lambda_{S R} \lambda_{R E}} \int_{0}^{\infty}\left\{1-2 \sqrt{\frac{\lambda_{S J} \lambda_{J E} w}{\gamma_{t h}}} K_{1}\left(2 \sqrt{\frac{\lambda_{S J} \lambda_{J E} w}{\gamma_{t h}}}\right)\right\} K_{0}\left(2 \sqrt{\lambda_{S R} \lambda_{R E} w}\right) d w
$$

\section{NUMERICAL RESULTS AND DISCUSSION}

The influence of $\psi$ on the system IP is plotted in Figure 3 with the main system parameters is set up as $\eta=0.8, \alpha=0.5, R=0.5,1.0,1.5 \mathrm{bps} / \mathrm{Hz}$ respectively. From the results, we can state that the asymptotic IP is convergence to the exact IP at the end of the $\psi$ values, and the analytical values are the same as the simulation values. The higher $\mathrm{R}$, the worst IP values.

The influence of $\psi$ on the system IP is presented in Figure4 with the main system parameters is set up as $\psi=15 \mathrm{~dB}, \alpha=0.25,0.5,0.85, \mathrm{R}=0.5 \mathrm{bps} / \mathrm{Hz}$ respectively. And the IP versus $\eta$ and Ris illustrated in Figures 5 and 6 .Here we set up the primary system parameters as $\psi=15 \mathrm{~dB}, \mathrm{R}=0.5 \mathrm{bps} / \mathrm{Hz}, \alpha=0.25,0.5,0.85$, $\eta=0.25,0.5,1$ respectively. From the results, we can state that the analytical values are the same as the simulation values. The better values of the IP is getting with the lower values of $\eta, \alpha$, but with the higher values of $\mathrm{R}$. All the research results are convinced by analytical expressions. 


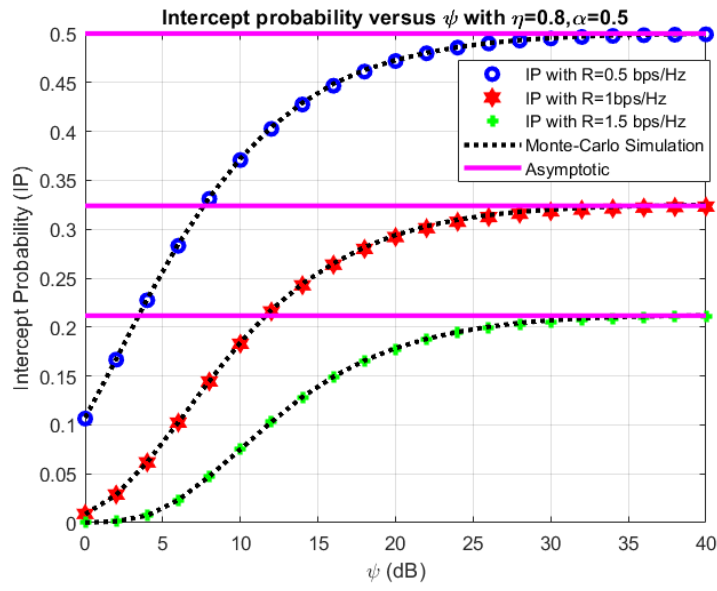

Figure 3. IP versus $\psi$

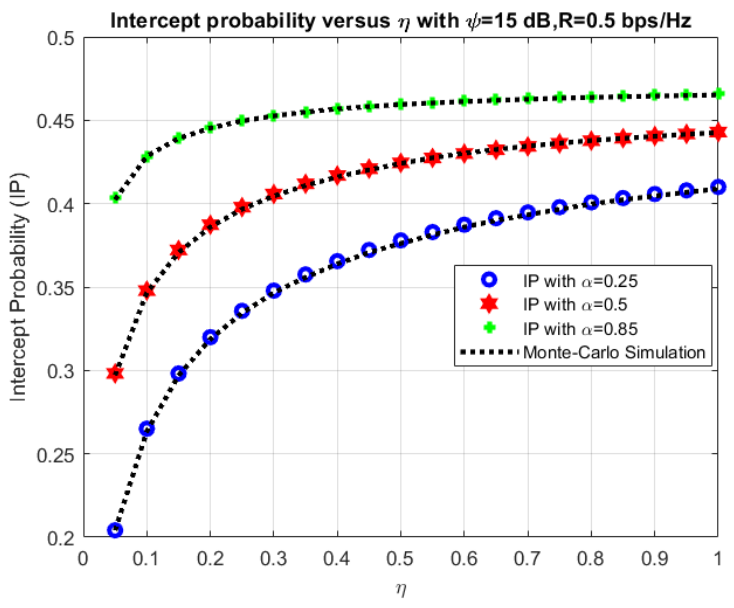

Figure 5. IP versus $\eta$.

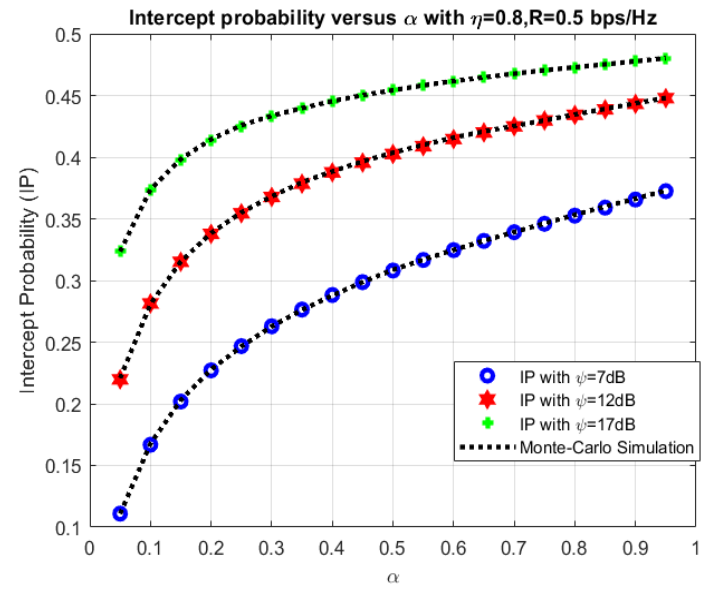

Figure 4. IP versus $\alpha$

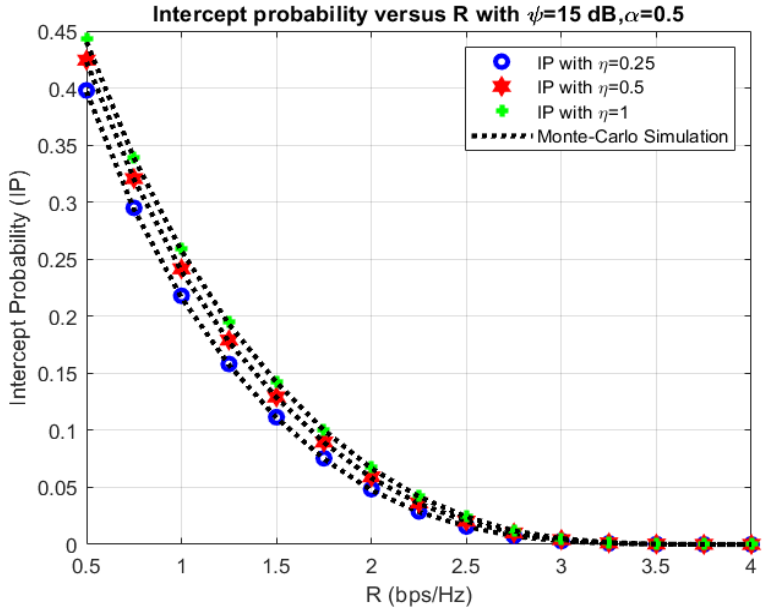

Figure 6. IP versus R

\section{CONCLUSION}

In thisresearch, the physical security layer with a friendly jammer in half-duplex (HD) relaying networks over the Rayleigh fading channel is proposed and investigated. In the first stage, we proposed the system model and the time switching, power splitting protocols for the system model. Then we conducted the mathematical analysis for deriving the exact analysis and asymptotic analysis integral forms for Intercept probability (IP). Finally, the analytical formulation is verified by the Monte Carlo Simulation with all main system parameters. The research results show that the analytical and simulation are the same values.

\section{ACKNOWLEDGMENTS}

This research was supported byNational Key Laboratory of Digital Control and System Engineering (DCSELAB), HCMUT, VNU-HCM, Vietnam.

\section{REFERENCES}

[1] S. Bi, C. K. Ho and R. Zhang, "Wireless powered communication: opportunities and challenges," in IEEE Communications Magazine, vol. 53, no. 4, pp. 117-125, 2015.

[2] D. Niyato, D. I. Kim, M. Maso and Z. Han, "Wireless Powered Communication Networks: Research Directions and Technological Approaches," in IEEE Wireless Communications, vol. 24, no. 6, pp. 88-97, 2017.

[3] Yu, H., Lee, H., \&Jeon, H., "What is 5G?Emerging 5G Mobile Services and Network Requirements,"Sustainability, vol. 9, no. 10, 2017. 
[4] Zhou, Xun, Rui Zhang, and Chin Keong Ho., "Wireless Information and Power Transfer: Architecture Design and Rate-Energy Tradeoff," in IEEE Transactions on Communications, vol. 61, no. 11,pp.4754-767, 2013.

[5] Tan N. Nguyen, T.H.Q.Minh, Phuong T.Tran, MiroslavVoznak, T.T.Duy, Thanh-Long Nguyen and Phu Tran Tin,"Performance Enhancement for Energy Harvesting Based Two-Way Relay Protocols in Wireless Ad-hoc Networks with Partial and Full Relay Selection Methods,"Ad hoc Networks, vol. 84, pp.178-187, 2019.

[6] Tan N. Nguyen, T.H.Q.Minh, Phuong T.Tran and MiroslavVoznak, "Energy Harvesting over Rician Fading Channel:A Performance Analysis for Half-Duplex Bidirectional Sensor Networks under Hardware Impairments," Sensors, vol. 18, no. 6, 2018.

[7] Tan N. Nguyen, T.H.Q.Minh, Phuong T. Tran and MiroslavVoznak, "Adaptive Energy Harvesting Relaying Protocol for Two-Way Half Duplex System Network over Rician Fading Channel," Wireless Communications and Mobile Computing, pp. 1-10, 2018.

[8] M. R. Bhatnagar, "On the Capacity of Decode-and-Forward Relaying over Rician Fading Channels," in IEEE Communications Letters, vol. 17, no. 6, pp. 1100-1103, 2013.

[9] Shannon, C. E., "Communication Theory of Secrecy Systems," Bell System Technical Journal,vol. 28, no. 4, pp. 656-715, 1949.

[10] Li, Bin, YulongZou, Jianjiang Zhou, Fei Wang, Weifeng Cao, and Yu-Dong Yao, "Secrecy Outage Probability Analysis of Friendly Jammer Selection Aided Multiuser Scheduling for Wireless Networks," IEEE Transactions on Communications, vol. 67, no. 5, pp. 3482-495, 2019.

[11] Alemdar, Ali, and Mohamed Ibnkahla, "A Survey of Wireless Sensor Networks: Technologies, Challenges, and Future Trends," Adaptation and Cross Layer Design in Wireless Networks, pp. 243-62, 2018.

[12] Y. Zhang, T. Liang and A. Sun, "Joint transmit antenna selection and jamming for security enhancement in MIMO wiretap channels," in IEEE/CIC International Conference on Communications in China (ICCC), pp. 1-6, 2015.

[13] Cumanan, et.al.,"Secrecy Rate Optimizations for a MIMO Secrecy Channel With a Multiple-Antenna Eavesdropper." IEEE Transactions on Vehicular Technology, vol. 63, no. 4, pp. 1678-690, 2014.

[14] P. Mukherjee and S. Ulukus, "Secrecy in MIMO Networks With No Eavesdropper CSIT," in IEEE Transactions on Communications, vol. 65, no. 10, pp. 4382-4391, Oct. 2017.

[15] Chu, Zheng, et.al., "Secrecy Rate Optimizations for a MIMO Secrecy Channel With a Cooperative Jammer," inIEEE Transactions on Vehicular Technology, vol. 64, no. 5, pp. 1833-847, 2015.

[16] Ju, E. Oh and D. Hong, "Catching resource-devouring worms in next-generation wireless relay systems: Two-way relay and full-duplex relay," in IEEE Communications Magazine, vol. 47, no. 9, pp. 58-65, 2009.

[17] Riihonen, Taneli, Stefan Werner, RistoWichman, and JyriHamalainen. Outage Probabilities in infrastructure-based Single-Frequency Relay Links. IEEE Wireless Communications and Networking Conference, 2009.

[18] D. W. K. Ng, E. S. Lo and R. Schober, "Dynamic Resource Allocation in MIMO-OFDMA Systems with FullDuplex and Hybrid Relaying," in IEEE Transactions on Communications, vol. 60, no. 5, pp. 1291-1304, 2012.

[19] I. Krikidis, H. A. Suraweera, P. J. Smith and C. Yuen, "Full-Duplex Relay Selection for Amplify-and-Forward Cooperative Networks," in IEEE Transactions on Wireless Communications, vol. 11, no. 12, pp. 4381-4393, 2012.

[20] S. Luo, R. Zhang and T. J. Lim, "Optimal save-then-transmit protocol for energy harvesting wireless transmitters," in IEEE International Symposium on Information Theory Proceedings, pp. 955-959, 2012.

[21] H. A. Suraweera, G. K. Karagiannidis and P. J. Smith, "Performance analysis of the dual-hop asymmetric fading channel," in IEEE Transactions on Wireless Communications, vol. 8, no. 6, pp. 2783-2788, 2009.

[22] SoukainaEssaghir, Mohamed Benchagra, Noureddine El barbri, "Comparison between PI and PR Current Controllers of a Grid- Connected Photovoltaic System Under Load Variation,"International Journal of Power Electronics and Drive Systems (IJPEDS), vol. 9, no. 3, pp. 1311-1320, 2018.

[23] Phu Tran Tin, Minh Tran, Tan N. Nguyen, Thanh-Long Nguyen, "System performance analysis of hybrid timepower switching protocol of EH bidirectional relaying network in amplify-and-forward mode," Indonesian Journal of Electrical Engineering and Computer Science, vol. 14, no. 1, pp. 118-126, 2019.

[24] Phu Tran Tin, Le Anh Vu, Tan N. Nguyen, Thanh-Long Nguyen, "User selection protocol in DF cooperative networks with hybrid TSR-PSR protocol based full-duplex energy harvesting over rayleigh fading channel: system performance analysis,"Indonesian Journal of Electrical Engineering and Computer Science, vol. 13, no. 2, pp. 534-542, 2019.

[25] Phu Tran Tin, Minh Tran, Tan N. Nguyen, Thanh-Long Nguyen, "A new look at energy harvesting half-duplex DF power splitting protocol relay network over rician channel in case of maximizing capacity,"Indonesian Journal of Electrical Engineering and Computer Science, vol. 13, no. 1, pp. 249-257, 2019. 